\title{
Del zine al podcast. Repensar la cultura de la participación desde un análisis comparativo de los medios alternativos
}

\section{From zine to podcast. Rethinking participatory culture from a comparative analysis of alternative media}

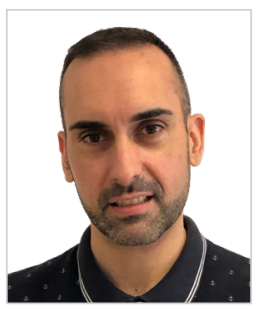

David García-Marín. Doctor en Sociología (especialidad Medios de Comunicación y Sociedad del Conocimiento) por la UNED. Profesor de Periodismo en la Universidad Carlos III de Madrid, donde imparte en inglés la asignatura Media Theory en los grados de Periodismo y Estudios Culturales. Paralelamente, ejerce como profesor invitado en diversos másteres en comunicación digital, nuevas pedagogías y periodismo transmedia en la UNED, donde además dirigió varios cursos sobre medios digitales y desinformación. Sus áreas de investigación se centran en el podcasting y el audio digital, la participación en medios digitales, la desinformación, el periodismo transmedia, y las pedagogías interactivas. Es autor y coordinador de numerosos artículos y libros centrados en las perspectivas actuales de la comunicación y el desafío de la desinformación en nuestra sociedad.

Universidad Carlos III, Madrid, España

dgmarin@hum.uc3m.es

ORCID: 0000-0002-4575-1911

Recibido: 21/11/2019 - Aceptado: 26/03/2020

\section{Resumen:}

La llegada de la Web 2.0 trajo consigo la explosión de numerosas teorías de la participación que vislumbraban el empoderamiento comunicacional ciudadano y la descentralización del relato mediático, ahora también potencialmente protagonizado por creadores no profesionales, "los antiguamente conocidos como la audiencia”. Este ensayo plantea un análisis comparativo entre el zine y el podcast como medios alternativos catalizadores del empoderamiento mediático y la distribución de discursos contraculturales, a partir del estudio de sus aspectos definitorios, tales como su financiación, distribución y circulación, la durabilidad de los proyectos, su carácter de medio personal, instrumental y social y sus lógicas creativas. A partir de este análisis, el trabajo teoriza sobre la falsa novedad de la cultura de la participación y las escasas posibilidades emancipatorias reales que los ciudadanos encuentran en los medios digitales, instando a un enfoque crítico hacia las utópicas visiones de la comunicación digital.

Palabras clave:

Podcasting; medios alternativos; participación; empoderamiento; zine.
Received: 21/11/2019 - Accepted: 26/03/2020

\section{Abstract:}

The advent of Web 2.0 entailed a surge of numerous participatory theories that heralded the advent of citizen communicative empowerment and the decentralization of the media discourse, nowadays potentially led by amateur creators, or "those who were formerly known as the audience". This essay poses a comparative analysis between the zine and the podcast as alternative media catalysts for media empowerment and the spread of counterculture narratives by studying their defining aspects, such as funding, distribution/circulation, project longevity, their features as media that is personalized, instrumental, and social, in addition to their creative rationale. From this analysis, the present study engages in a theoretical inquiry into the false novelty of participatory culture and the few possibilities of liberation that citizens can find in digital media, urging a discerning approach to utopian visions regarding digital communication.

Keywords:

Podcasting; alternative media; participation; empowerment; zine.

Cómo citar este artículo:

García-Marín, D. (2020). Del zine al podcast. Repensar la cultura de la participación desde un análisis comparativo de los medios alternativos. Doxa Comunicación, 30, pp. 107-125.

https://doi.org/10.31921/doxacom.n30a5 


\section{Introducción}

Las primeras aproximaciones a la llamada cultura de la participación surgen a partir de la llegada de los instrumentos digitales e interactivos propios de la Web 2.0. Las diferentes teorías de Gillmor (2004), Benkler (2006), Rheingold (2000), Shirky (2010), Castells (2012), Jenkins, Green y Ford (2015) y Jenkins, Ito y Boyd (2016) subrayan el empoderamiento comunicacional adquirido por los sujetos; a la vez que defienden la descentralización del relato mediático, ahora también teóricamente en manos de los públicos. En este contexto, el podcasting, por su facilidad de producción y distribución de contenidos a una audiencia potencialmente mundial, es uno de los medios digitales que, en línea con estas teorías, capacita a los individuos como potenciales participantes en el discurso público junto a los medios de comunicación de masas que durante el siglo XX monopolizaron la información y, con ella, las formas de representación de la realidad.

El podcasting surgió en Estados Unidos en 2004 como un nuevo medio de comunicación basado en la distribución de contenidos sonoros a través de la Web mediante sistemas de sindicación que permiten la suscripción de los usuarios. Los pioneros de este medio fueron Adam Curry, un antiguo videojockey del canal televisivo MTV y Dave Winer, uno de los desarrolladores del motor de sindicación de contenidos RSS que ya funcionaba en páginas web y blogs. Ambos fueron capaces de construir un método de emisión de audio vía web que pone los archivos sonoros a disposición de cualquier oyente para ser escuchados a su conveniencia. El primer podcaster español, José Antonio Gelado, procedía del mundo de la radio y su salto al podcasting se fundamentó en la búsqueda de un modo para continuar su labor radiofónica con una mayor dosis de libertad bajo la experimentación con el audio por suscripción. Como otros pioneros del medio, Gelado disponía de un blog y, como ya permitían estas bitácoras online, su objetivo era ofrecer contenido de audio de forma sindicada que rompiera las rígidas estructuras de la radio.

Si la radio es un medio paradigmático de la era de los mass media, el carácter digital 2.0 del podcasting promueve la forma de escucha de productos sonoros bajo varias lógicas disruptivas que diferencian claramente a ambos medios. Los podcasts presentan una mayor variedad temática que se ve claramente reflejada en el podcasting amateur, cuyos creadores abrigan sin complejos la libertad de creación de contenidos abordando temas que habitualmente se quedan fuera del circuito mainstream. De este modo, podemos encontrar programas de la temática más variopinta que sitúan al medio en una búsqueda constante del nicho disponible para ser ocupado. Bajo este prisma encontramos una de las quiebras del podcasting con respecto a la radio hertziana. Si ésta es un medio que se acerca más al modelo de consumo hit-caracterizado por la provisión de un número limitado de materiales a unas audiencias masivas bajo un modelo de-uno- $a$ muchos-debemos situar al podcasting como una práctica que atomiza a sus audiencias a partir de la difusión de contenidos mucho más específicos. Aunque, es necesario matizar que, en ciertos contextos como el norteamericano, la radio ha adquirido cierto nivel de segmentación de contenidos gracias, en parte, a la proliferación de emisoras que actúan en ámbitos locales y al desarrollo de proyectos radiofónicos digitales focalizados en temáticas específicas, sobre todo dentro del campo musical. Estas atomizadas formas de escucha, establecidas de forma nativa en el podcasting, se vinculan con la lógica long tail (larga cola), un modelo que distribuye menos cantidad de un mayor número de productos; es decir, muchos programas de variado contenido para un público minoritario y específico. El podcasting, por tanto, diversifica la oferta de contenidos que podemos encontrar en el ecosistema mediático, ofreciendo materiales culturales difíciles de 
conseguir a través de otras vías. De forma análoga, este medio no solo provee contenidos alternativos, sino que pone a disposición enfoques diferenciados con respecto a temas que ya están instalados en la agenda-setting, ejerciendo como motor extracultural y alternativo a los relatos de los medios hegemónicos.

La flexibilidad y la personalización de la escucha también son aspectos diferenciales del podcasting frente a la radio. La posibilidad de operar sobre las condiciones de recepción del contenido gracias a sus características de automatización y digitalización abren a los podcasts nuevos campos interactivos desconocidos para la radio. Igual que cada internauta realiza un recorrido diferente a través de la Web, los oyentes de podcasts disfrutan de múltiples maneras de relacionarse con el contenido sonoro, que van desde la escucha completa de principio a fin, la continua interrupción de la reproducción del audio, los saltos hacia adelante y hacia atrás o la búsqueda de las secciones de cada programa más interesantes para el usuario. En el podcasting las posibilidades de consumo mediático se multiplican frente a la escucha lineal, estandarizada y homogénea de la radio. La interacción usuario-medio se vuelve flexible, personalizada, adaptada y de linealidad múltiple.

Este modelo que empodera al sujeto en el proceso de recepción y consumo de contenidos provoca que dirijamos nuestra mirada hacia la idea del compromiso frente a las antiguas lógicas del comisariado, que permanece anclado en las relaciones verticales entre programadores y seguidores de los productos culturales, obligados a consumir el contenido en el día y a la hora concreta que tales programadores deciden y a través de las plataformas impuestas por éstos. Frente a este sistema -muy presente en los medios tradicionales- se eleva el modelo del compromiso que destruye las relaciones jerárquicas entre franquicia y fans. Contraponiendo el modelo del compromiso frente al del comisariado, Jenkins, Ford y Green (2015) afirman que allá donde haya un medio que haga partícipe a sus seguidores, habrá usuarios comprometidos con su escucha y difusión, propagación y recomendación a través de la Red.

\section{Estudios sobre el podcasting. Antecedes empíricos y teóricos}

La tesis doctoral de Sellas (2011) constituyó el primer estudio sistemático sobre el podcast en el contexto español. Sus investigaciones concluyeron que este medio conlleva un cambio profundo en la forma de comunicación sonora al plantear nuevas posibilidades frente a la radio tradicional y por reunir a un conjunto de creadores independientes caracterizados por su libertad, espontaneidad e informalidad sin estar condicionados por las rutinas de producción y programación de la radio, de modo que pueden contribuir a renovar el estilo y el lenguaje de la comunicación sonora.

Gallego (2010), desde una perspectiva mercadotécnica, centra su mirada en la relación entre la empresa radiofónica y el podcasting analizando la repercusión de este medio en el modelo de negocio de las radios convencionales y cómo éstas utilizan las múltiples posibilidades que el podcast ofrece. García-Marín (2017) aborda el podcast desde el ámbito narrativo, situándolo como un claro ejemplo de medio transmedia (transpodcast) que, de forma análoga al concepto de transradio de Martínez-Costa (2015), desborda el lenguaje exclusivamente sonoro de sus inicios y extiende sus relatos a otras plataformas que contribuyen a complementar los contenidos y buscar la participación de los usuarios que, de este modo, se convierten en protagonistas de la podcastfera, comunidad caracterizada por la construcción de un complejo ecosistema (García-Marín, 2019) de oyentes y creadores -no profesionales en su mayoría- donde cohabitan podcasts 
independientes, redes profesionales y amateurs y una creciente producción de trabajos procedentes del ámbito mediático profesional, sobre todo desde los operadores de radio tradicionales (Pérez-Alaejos, Pedrero-Esteban y Leoz Aizpuru, 2018). Asimismo, gran parte de la atención investigadora hacia el medio en los últimos años en el contexto español se centra en las posibilidades del podcast como herramienta educativa y su efectividad en el aprendizaje de materias como los idiomas (Ortega, 2019), la historia de la música (García-Peinazo, 2019) y la divulgación de la ciencia (De Lara-González y Del Campo-Cañizares, 2018).

El paisaje académico en el panorama anglosajón presenta investigaciones realizadas desde puntos de vista muy diversos. Markman (2011) desarrolló un análisis exploratorio sobre el perfil de los podcasters independientes. Un perfil definido por hombres con edades comprendidas entre los 34 y los 44 años, con una elevada formación y con profesiones ubicadas en los campos de las artes, el diseño, el entretenimiento, los medios, la informática, la tecnología y la educación. Según su estudio, los motivos que encuentran estos creadores para iniciar su labor en el podcasting se dividen en: factores mediáticos y tecnológicos (por ejemplo, haber tenido experiencia previa en otros medios y, sobre todo, en el campo de la radio), relacionados con el contenido (llenar un nicho que los medios tradicionales no ocupan) y motivaciones personales e interpersonales (unirse a un movimiento o comunidad). A estas motivaciones, Sullivan (2018) añade el trabajo aspiracional, es decir la esperanza de iniciar una práctica amateur con la expectativa de futuros beneficios económicos. Millette (2011) centró también sus estudios en los podcasters independientes, capaces de construir un medio que supone una auténtica discontinuidad con respecto al modelo radiofónico convencional. Su estilo es conformado a través de un singular nivel de familiaridad entre usuarios y podcasters y la inclusión de un léxico disruptivo frente a las convenciones de la radio. Desde esta óptica, el podcasting independiente es una de las subculturas participativas que han emergido con el auge de las herramientas y las prácticas 2.0, desplegada alrededor de un uso específico de Internet que sirve como forma de expresión para el podcaster y que existe en tensión con la cultura radiofónica tradicional. El carácter participativo del medio puede llegar a tener una dimensión performativa, implicando la interacción física del oyente con el espacio descrito en el podcast (Wilson, 2018).

Berry (2018) y Spinelli y Dann (2019) subrayan que el podcasting debería ser percibido como un medio con identidad propia, no como una extensión de otras prácticas mediáticas. En una línea similar, Massarelli y Perrotta (2006), Cordeiro (2012) y Bonini (2015) abordaron el podcasting como una tecnología desafiante para los medios tradicionales, al permitir a los usuarios crear su propio contenido, a la vez que constituye un cambio en los hábitos de escucha de la radio convencional. Lindgren (2014) defiende que el podcasting constituye un modo ideal para la construcción de narrativas en primera persona, incluso en su aplicación al campo del periodismo. El movimiento hacia este tipo de narrativas estaría intrínsecamente ligado a la naturaleza íntima de los medios sonoros que, utilizando diferentes géneros y formas, explotan esta manera personal de contar historias.

Los estudios de McHugh (2016) se centran en el impacto del podcasting en los formatos sonoros no ficcionales. Sus trabajos analizan el rol cambiante asumido por los medios públicos, el crecimiento de redes de podcasts independientes y las diferencias percibidas entre la radio y el podcasting como modos de distribución y recepción de contenidos sonoros. En la misma línea iniciada años antes por Millette, McHugh defiende que el podcasting fomenta un género novedoso y más informal de narrativa sonora centrada en unas fuertes relaciones entre los presentadores y los oyentes, con contenidos 
más improvisados y menos elaborados. Para este autor, uno de los grandes retos del sector del podcasting estadounidense es la provisión de mayores recursos sin comprometer su independencia editorial, problemática que permanece aún sin resolver.

Desde otro punto de vista, la capacidad conectiva del podcasting es analizada en los estudios de Swiatek (2018) al poner de manifiesto el potencial relacional del medio, capaz de cerrar brechas socio-culturales y de conocimiento. Berry (2015) pone su atención en la primera década de desarrollo del podcasting examinando el caso de Serial, el podcast más exitoso en cuanto a volumen de descargas de la breve historia del medio. Desde su inicio, un elemento clave del podcasting ha sido la baja importancia que creadores y escuchas le han dado a la calidad técnica de los programas, en comparación con la radio. De acuerdo con Berry, el ejemplo de Serial, un programa dotado de elevados recursos técnicos, pudo contribuir a elevar los estándares de calidad del medio a fin de llegar a nuevos oyentes (Hancock y McMurtry, 2018). El éxito de Serial provocó una renovada atención hacia el podcasting y fue capaz de atraer a nuevos consumidores (Sellas y Solá, 2019). Wrather (2016) analiza cómo los usuarios de este medio son dirigidos hacia espacios online diferentes al texto central del podcast a fin de potenciar su relación, compromiso y participación en el seno de los programas. Su trabajo proporciona una cartografía de las diferentes estrategias interactivas entre podcasters y oyentes analizando la actividad de ambos en los espacios sonoros, foros de Internet y redes sociales de los programas, en un medio considerado claramente user-centered donde el oyente debe decidir cómo ejecuta su consumo e interacción ya que, a diferencia de otros medios, en el podcast no existe una única forma posible (Llinares, 2018).

Como observamos, a pesar de la abundancia de investigaciones y producciones teóricas alrededor del medio, existe un claro déficit de miradas hacia las posibilidades contraculturales del podcast y sus potencialidades para un verdadero empoderamiento comunicacional del ciudadano. Por ello, es objeto de este trabajo teórico establecer líneas de conexión entre los medios alternativos analógicos -especialmente los zines que proliferaron a finales del siglo XX-y el podcasting, a fin de establecer una cartografía de los elementos que, bajo procesos de remediación (Bolter y Grusin, 2000), conecten los viejos con los nuevos medios a partir de su dimensión participativa, alternativa y disruptiva, en línea con las teorías de la participación y el empoderamiento ciudadano surgidas al calor de la Web 2.0.

\section{Los medios alternativos}

En su libro Why study the media?, Roger Silverstone (1999) define los medios alternativos como aquellos capaces de crear nuevos espacios para voces diferentes que ponen en el centro de su acción intereses comunitarios específicos así como aspectos subversivos con respecto a la cultura dominante, poniendo de manifiesto el empleo de técnicas propias de los medios de masas para perseguir una agenda crítica o disruptiva desde los márgenes. Esta definición parece, no obstante, insuficiente, ya que los medios que desarrollan lógicas contraculturales o buscan introducir aspectos no tratados en los circuitos comerciales no agotan la lista de candidatos para ser denominados como medios alternativos. Los editores de Alternatives in Print (1980), considerado como uno de los mejores trabajos bibliográficos en el campo de la comunicación alternativa, presentan tres criterios elementales para la definición de este tipo de medios: los proyectos obligatoriamente no han de tener carácter comercial y, por tanto, deben mostrar una preocupación fundamental por las ideas, 
no por el beneficio; y la materia de su publicación debería centrarse en la responsabilidad social, la expresión creativa o una combinación de ambas. Nuevamente, esta definición nos dirige hacia conceptualizaciones conflictivas. El segundo aspecto presenta dificultades por la práctica imposibilidad de demostrar la prevalencia de las ideas frente al beneficio o viceversa. Además, podemos encontrarnos medios cuya misión sea el mantenimiento del orden establecido -por tanto, muy preocupados por la ideología- que no encajarían con una visión emancipatoria del uso de la comunicación, sino por todo lo contrario. Asimismo, Atton (2002) matiza que la inclusión en el tercer aspecto del criterio de "expresión creativa o una combinación de ambos" ensancha en demasía la definición de medios alternativos de modo que cualquier tipo de publicación artística podría estar incluida bajo tal denominación.

Referido anteriormente, Chris Atton dedicó parte de su producción investigadora al análisis de los zines, publicaciones escritas desarrolladas como vehículos para la expresión personal que mantienen procesos comunicativos horizontales entre editores y lectores y cuyo formato anima precisamente a estos últimos a convertirse en autores. Según Duncombe (1997), la emulación -convertir a los lectores en escritores- es elemental para el mundo del zine; la cultura es mejor cuando más consumidores son capaces de convertirse en productores, es decir que los lectores o espectadores pasen a jugar el rol de colaboradores significativos. El término zine fue introducido en los años 80 para denominar a un amplio rango de publicaciones amateurs, entre las que destacaban los fanzines, que ponían su foco primariamente en contenidos como la literatura, la música, el cine y otras actividades culturales, aunque no solo se referían a prácticas de consumo (Atton, 2002). De hecho, Fiske (1991) argumenta que los creadores de fanzines deben ser considerados como productores culturales, no consumidores de cultura: en el corazón de la cultura del zine no está solo el estudio del otro (la celebridad, el objeto cultural o la actividad), sino el estudio del yo, la expresión personal, así como la construcción de comunidades de afinidad.

Estos principios fundamentales que establecieron las lógicas creativas y la distribución mediática en el mundo de los zines se muestran claramente presentes en el podcasting, medio que, de este modo, recupera -remedia- elementos esenciales de los medios alternativos de la era analógica. Bolter y Grusin (2000) afirman que los procesos de remediación (la introducción de un medio en otro medio o la transferencia de características propias de medios antiguos en nuevas especies mediáticas) no comienzan con la llegada de los medios digitales. La tesis sobre la que se asienta la obra de estos autores defiende que ningún medio actual -y posiblemente ningún medio futuro- funciona en ausencia de otros medios y que los aspectos realmente novedosos de los llamados nuevos medios son las formas en que los antiguos son remodelados para responder a nuevos desafíos. Bajo este prisma, las nuevas especies mediáticas que han surgido a lo largo de la historia supondrían la evolución, mejora o sofisticación de algunos de los fundamentos de manifestaciones mediáticas anteriores. Desde su punto de vista, los nuevos medios han de ser entendidos como constructos que desbordan los límites de la tecnología al suponer mucho más que nuevas aplicaciones técnicas al servicio de los ciudadanos. Los nuevos medios emergen desde contextos culturales concretos y constituyen la suma de nuevos usos y protocolos en situaciones y ámbitos específicos, tales como la publicidad, la educación o la expresión personal. Desde esta visión, el concepto de nuevo medio resultaría una categoría problemática a la hora de definir las nuevas manifestaciones y prácticas mediáticas, de forma que éstas no existirían como tales, sino que solo serían una potenciación de las características de medios 
anteriores. Bajo este prisma, lo único realmente nuevo de los nuevos medios es su incesante remediación de otras formas de mediación.

En este sentido, ¿cuáles son las remediaciones que podemos encontrar entre los zines y los actuales podcasts? ¿Qué elementos de los primeros son recuperados por el podcasting para que éste pueda ser definido como medio alternativo? Ambos medios, cuyos puntos de partida están separados por prácticamente medio siglo, presentan una naturaleza claramente diferenciada en términos tecnológicos: el zine presenta una base analógica frente a la naturaleza digital nativa del podcast. También difieren en cuanto a sus lenguajes de producción: el zine está basado en creaciones textuales e imágenes estáticas, mientras que la base del podcast es sonora. La distancia cronológica de sus momentos de aparición, así como sus diferencias tecnológicas y semióticas, justifican el análisis de las remediaciones entre ambos medios. El planteamiento de un estudio de este tipo en medios similares puede resultar menos relevante porque tales remediaciones son más evidentes y porque este tipo de trabajos son más habituales, como sucede en los estudios que relacionan la radio (analógica o digital) y el podcast, véase el caso de Ramos-Ruiz (2015), Espada (2017), Rodríguez-Pallarés (2017), López-Villafranca (2019), Moreno-Espinosa y Román-San Miguel (2020).

Como se indicaba anteriormente, en este ensayo, se pretende mostrar la existencia de una continuidad en términos de producción, alcance, objetivos y papel del usuario entre dos medios alternativos claramente diferenciados. Sostenemos que, a pesar de la distancia ontológica y semiótica de ambos medios, existe una línea común en términos de participación y empoderamiento comunicacional del ciudadano que impugna las perspectivas que defienden que la participación en los medios va ligada a la explosión de lo digital. Las posibilidades de empoderamiento comunicacional del ciudadano no han llegado con el mundo digital. Ciertas formas participativas e intercreativas entre usuarios y medios ya estaban presentes en medios alternativos predigitales. Es por ello que los medios participativos no existen por sí mismos. Lo que existen son los sistemas de participación (dependientes, por tanto, de una multiplicidad de factores) que cada proyecto mediático es capaz de configurar, independientemente de la categoría mediática donde se les pueda etiquetar. Por ello, en lugar de centrar nuestro foco en un único aspecto, abordamos el análisis de ambos medios desde una perspectiva holística y sistémica a partir del modelo de Atton (2002) descrito en Alternative Media, obra de referencia -enfocada en los zines- en el análisis de los medios alternativos. Siguiendo su modelo, realizamos una observación analítica sobre la presencia tanto en el zine como en el podcasting de las siguientes dimensiones: su financiación y alcance, la durabilidad de los proyectos, su carácter de medio personal, su capacidad instrumental y, finalmente, la amplitud de estrategias creativas y su carácter experimental (lógica IDIC).

\section{Remediaciones zine-podcasting}

\subsection{Financiación y alcance}

El podcasting, al igual que sucedía de forma amplia en la cultura del zine, se encuentra lejos de monetizar su producción y obtener beneficios, puesto que la inmensa mayoría de proyectos son claramente amateurs, incluso mantienen una mayor ausencia de capitalización que la encontrada en muchos otros proyectos alternativos. Por ejemplo, en el caso de los 
zines, el modelo de suscripción era un método común a la hora de obtener financiación. Esta entrada de capital generaba al menos una pequeña seguridad financiera, ofreciendo la posibilidad de realizar cierta planificación en la actividad del medio. Para el mantenimiento de los proyectos en el podcasting, sin embargo, la adquisición de recursos económicos no resulta tan crucial como sí lo era para el sustento de los zines, ya que éstos debían correr con mayores gastos al no disponer de una base digital para el desarrollo de sus operaciones. A pesar de ello, los zines representaban una forma de comunicación relativamente barata gracias a las tecnologías reprográficas que ejercían el papel de las grandes rotativas de los periódicos masivos. Durante los años 70 y 80, los medios alternativos utilizaron ampliamente las máquinas reprográficas, que les hicieron, sino financieramente independientes, al menos sí técnicamente.

En el podcasting, esta ausencia de capitalización se ve favorecida por la práctica nula necesidad de inversión a la hora de iniciar un proyecto. El uso de servicios digitales gratuitos, el abaratamiento progresivo de los instrumentos de audio necesarios (micrófono y mesas de mezclas) y la gratuidad que ofrece Internet para la distribución de contenidos ofrecen unas facilidades desconocidas para los productores de los medios alternativos analógicos. Esta facilidad de producción y distribución favorece la constitución del podcasting como un medio de nicho: si cualquier ciudadano puede sumarse a la comunidad de creadores desde los márgenes de la agenda de los grandes medios tradicionales, la diversidad temática y de enfoques está prácticamente asegurada, si bien el número de seguidores de estos proyectos mediáticos no sea excesivamente elevado.

Sin embargo, la evolución de determinados proyectos de podcasting inicialmente amateurs hacia posiciones más comerciales ha llevado a ciertos creadores a poner en práctica diversas estrategias de monetización de sus proyectos. A la inserción de publicidad y las iniciativas de crowdfunding se añaden modelos de transmedialización de los podcasts donde los contenidos sonoros se complementan con programas en directo como si de obras teatrales se trataran, con acceso previo pago. Otra estrategia comúnmente seguida en el mundo amateur es la patreonización de las producciones. El modelo obtiene su nombre del servicio Patreon, plataforma de micromecenazgo que permite a creadores de contenido digital obtener recompensa económica por su labor. Determinados podcasts capaces de generar un amplio volumen de contenido en diferentes plataformas y lenguajes mediáticos establecen planes de suscripción a sus producciones de modo que existe un paquete mínimo para aquellos usuarios no suscritos, y contenido exclusivo para los seguidores que apoyan económicamente el proyecto. Es el caso de ciertas redes de podcasts procedentes del mundo amateur, como la española Emilcar FM, creadas antes de la llegada en 2016 de las redes de podcasts profesionales al contexto español con el lanzamiento de Podium Podcast. Esta división entre usuarios se establece también desde el lado de la participación significativa: determinados proyectos, tales como la red independiente Nación Podcast, abren sus puertas a la cocreación para los usuarios que mejores aportaciones económicas realizan, incorporando a tales seguidores como invitados con participación activa en sus grabaciones.

En este punto, debemos situarnos en el debate sobre el alcance y seguimiento de estos medios. La comunidad de creadores en el podcasting está configurada por una enorme multiplicidad de proyectos, con variadas temáticas pero con un número de seguidores y usuarios poco significativo en comparación con las cifras que manejan los grandes medios nacionales, e incluso, regionales. $i$ Esta falta de volumen de seguimiento, de tamaño, debe ser considerada como un fracaso o, al menos, como una dificultad? Existen visiones contrapuestas en cuanto a la necesidad de alcanzar a un número masivo de 
ciudadanos para que los medios alternativos puedan ser considerados realmente útiles: frente a la opinión que defiende un ecosistema con pocos medios alternativos muy grandes, se sitúan aquellos que abogan por la diversidad, es decir la existencia de un alto número de proyectos aunque éstos no alcancen un tamaño reseñable.

Entre aquellos que defienden la dimensión frente a la diversidad se sitúa Aubrey (1981), quien afirma que, colocados en la tesitura de elegir entre una alta circulación de un puñado de cabeceras o la pequeña circulación de un diverso o ancho rango de éstas, es preferible la primera situación. Como argumento de su tesis, afirma que el relato de los medios alternativos que adquieren cierto tamaño puede verse diluido por la aparición de numerosos medios más pequeños que traten una infinidad de temas que fragmentan y debilitan su discurso.

Ante esta opinión, se sitúan los creadores del zine Bypass, quienes defienden que cientos de medios con poca circulación no solo animan la diversidad de información y opinión, sino que aseguran la supervivencia de la prensa alternativa. Del mismo modo, desde el Oxford Institute of Social Disengineering, consideran la proliferación de publicaciones como una fortaleza democrática, de forma que "un modelo de cien medios con un alcance de mil usuarios tiene mejores consecuencias que una sola publicación con un impacto de 100.000” (Atton, 1996).

De acuerdo con Ilich (2002), determinadas instituciones sociales, una vez que alcanzan cierta dimensión e importancia, pueden causar más perjuicio que beneficio. En este sentido, pone como ejemplo la escuela que, convertida en un sistema que provee educación conforme a unas reglas determinadas así como la memorización de un conjunto de conocimientos sin el necesario aprendizaje o entendimiento, es considerada como un fin en sí misma, no como una herramienta para el ciudadano. Por consiguiente, según la tesis de Illich, la institución escolar hace a la gente estúpida, del mismo modo que la institución médica convierte a los ciudadanos en enfermos y el sistema de negocios mundial arruina el planeta (Gauntlett, 2011). Estableciendo una analogía, podemos inferir que, bajo este mismo prisma, un sistema concentrado con solo un puñado de grandes medios de comunicación (es decir, pocos medios pero con mucho tamaño) convierte a los ciudadanos en desinformados y alejados del entendimiento del mundo. A este respecto, el modelo que plantea el podcasting como fuerza alternativa facilita la creación de un discurso propio desde la actividad mediática ciudadana que bombardea la lógica vertical de la masiva influencia aplicada por las grandes instituciones. Aunque en los últimos años y gracias a la popularización del medio, determinados podcasts independientes y con estética amateur se han convertido en proyectos con un seguimiento masivo y han devenido en producciones claramente comerciales, el podcasting representa un espacio donde la creatividad personal y colectiva del Do It Yourselfy, sobre todo, del Do It Together (hazlo con otros) pone en circulación interpretaciones alternativas de la realidad mediante procesos que privilegian el modelo de la amplia circulación y sobreabundancia de medios de tamaño menor. En palabras de Gauntlett (2011), debería ser desarrollado un conjunto de aproximaciones pequeñas, específicas y locales centradas en las necesidades del ciudadano, en detrimento de las grandes operaciones burocráticas. El propio Gauntlett propone el término conviviality para designar un tipo significativo de comunicación y compromiso entre ciudadanos activamente conectados y que mantienen una actitud viva con respecto a la realidad, constituyendo un vehículo para la formación de su propio mundo. La sociedad necesitaría, por tanto, herramientas para la creatividad individual, a fin de capacitar al ciudadano la realización de su propia vida desbordando las instituciones que tienden a imponer modelos de estandarización masiva. 


\subsection{Durabilidad}

La apertura de Internet para asentar proyectos mediáticos fáciles de distribuir y la amplia disponibilidad de herramientas sencillas y baratas para construir los mensajes abren las puertas de la producción mediática online a muchos ciudadanos que poco o nada tienen que ver con la comunicación profesional. A la vez, el carácter amateur no remunerado de la inmensa mayoría de los proyectos de podcasting facilita la escasa durabilidad de tales programas, muy dependientes de los avatares personales y profesionales de sus productores. Esta es una característica común de los medios alternativos que, a pesar del aparente perjuicio que puede representar para establecer proyectos que permanezcan en el tiempo y puedan tener cierto seguimiento, se ha considerado como un aspecto ventajoso en determinadas circunstancias. Bey (1991) y Labelle (2018) defienden la naturaleza transeúnte de estos medios de resistencia argumentando que esta característica constituye una ayuda para evitar que el poder les identifique y neutralice sus actividades. Entiéndase este argumento en el seno de aquellos medios que tengan un verdadero carácter contestatario y realicen una labor claramente crítica hacia el establishment. Por otro lado, la escasa durabilidad de estos proyectos sirve para evitar que algún medio pueda ganar autoridad sobre los demás, de modo que la falta de permanencia de determinados zines era, incluso, celebrada por la comunidad, en un claro movimiento que privilegia la lucha o las ideas que tales medios defendían en detrimento de la importancia del medio concreto en sí mismo.

En esta cuestión, sin embargo, no existe un claro consenso. La visión de McKay (1996) incorpora la idea de constancia transgresora (transgressive constancy), que acentúa la necesidad de efectividad y deseo de transformación permanente a fin de desbordar el simple deseo de transgresión, por lo que sería deseable y necesaria la presencia de proyectos mediáticos alternativos perdurables a largo plazo.

En el podcast independiente, la ausencia de durabilidad de los proyectos se relaciona, además, con las formas específicas en las que sus creadores adquieren las competencias necesarias para la construcción de este tipo de proyectos. Antes de lanzarse a la creación de trabajos de mayor envergadura, los podcasters amateurs lanzan programas de pequeño formato para adquirir las habilidades que requiere esta práctica mediática. Estos espacios (el podcast sobre arquitectura $L a$ morsa era yo es un ejemplo) funcionan como campos de prueba bajo principios de ensayo-error, por lo que habitualmente suelen ser abandonados cuando sus productores deciden aventurarse en la realización de producciones de mayor complejidad.

\subsection{Medio personal}

A pesar del elevado volumen de trabajo que es necesario afrontar para sacar adelante un proyecto de podcasting (es necesario recordar que este medio no solo implica labores en cuanto a la construcción de mensajes sonoros, también provee a la comunidad de seguidores un conjunto de extensiones narrativas en otros formatos y lenguajes, con el consiguiente esfuerzo que ello conlleva), existen podcasts transmedia que son diseñados y desarrollados de principio a fin por una sola persona que de forma individual se muestra presente en todas las fases del proceso de producción y distribución de los contenidos. Ciertos programas de esta naturaleza personal (ejemplos relevantes de este tipo de podcasts en el ámbito hispanohablante son Un minuto en Nueva York, Madrillano Podcast, Carmenia en Dallas -convertido en Carmenia en 
casa durante la cuarentena del COVID-19- y Haciendo el sueco) suelen abordar los aspectos de la vida diaria del productor, bien relacionados con la experiencia del podcaster a propósito de un tema más concreto (por ejemplo, su visión sobre la tecnología puesta a la luz de su relación diaria con determinados dispositivos, aplicaciones o software), o bien por la naturaleza ciertamente particular del periplo vital del creador (ciudadanos en el extranjero que narran su día a día a modo de guía para aquellos que pretendan viajar a tales países).

Esta auto-narrativa es también uno de los principios diferenciales de los medios alternativos analógicos. Perzines (personal zines) como Cometbus pueden ser considerados como ejemplos de producción popular enraizada en las especificidades de la vida cotidiana; medios en los que los autores proyectaban su propia personalidad en el abordaje de prácticas culturales, presentando sus propias vidas como contenido en sí mismo (Lefebvre, 1991). Con esta práctica, estos productores crean medios con una personalidad tan marcada que les sirven para construir identidades y relaciones sociales.

\subsection{Medio instrumental y social}

La creación de identidad y el establecimiento de relaciones no dejan de ser consecuencias instrumentales vinculadas al establecimiento de las prácticas de autoexpresión que observamos en el podcasting que, en este sentido, se configura como un medio no solo para comunicar con él, sino para hacerlo a través de él y mediante la creación de una vasta comunidad de productores y usuarios enlazada con ataduras virtuales y eventos presenciales, tales como las Jornadas Nacionales de Podcasting (JPod) y encuentros similares de menor ámbito geográfico. En el año 2019, los Podcast Days, evento promovido desde la asociación madrileña de podcasting, tomó el relevo de las JPod (que se celebran anualmente en diferentes ciudades desde su nacimiento en 2009 en Murcia) como principal evento que reúne a productores y entusiastas del medio en el ámbito hispanohablante. Estas iniciativas integran tres tipos de actividades que contribuyen al mantenimiento y desarrollo de la propia comunidad: talleres formativos de componente eminentemente práctico, ponencias y mesas redondas de tipo teórico y reflexivo sobre el estado del arte y programas en directo. Para su celebración, resulta fundamental el concurso de la Asociación Podcast que, nacida en 2010, provee soporte legal al equipo organizador de cada una de las ediciones.

El podcasting, a través de la construcción de redes que configuran una sólida comunidad, se muestra como un instrumento para alcanzar propósitos que se sitúan más allá de los meros objetivos comunicativos, informativos, culturales, identitarios o transformadores. La instrumentalidad de los medios alternativos, al menos en el sentido de la búsqueda de contactos personales, posibilidades de viajar o la adquisición de bienes, no es solo restringida a la cultura punk, donde se desarrolla con mayor profusión. Sarah Lorimer (editora de dos zines, Pinto y Baby I Dig You), mencionada por Atton (2002), sugiere que sus motivos para la publicación incluyen tanto el hecho de compartir reflexiones personales como un amplio rango de fines de tipo instrumental donde la interacción comunitaria y el funcionamiento en red de sus miembros ejercen un papel primordial.

En el mismo sentido, tras década y media de evolución, la podcastfera española puede ser considerada como una red comunitaria constituida por creadores mediáticos y usuarios caracterizada por un conjunto de fundamentos que le otorgan una identidad propia. Hemos de entender esta comunidad como la amalgama de espacios tanto online como offline 
compartidos por los productores de proyectos de podcasting y los usuarios y fans de tales producciones, independientemente del número de creaciones realizadas -en el caso de los podcasters- y del grado de participación y compromiso, en el caso de los usuarios. Las interacciones, conexiones, conversaciones, debates y proyectos conjuntos que se desarrollan alrededor de este medio contribuyen a coser una comunidad definida como un espacio de creadores Do It Yourself, como un mecanismo de socialización intermedia que conecta la producción individual con la conexión social, como una comunidad de código abierto, como un lugar donde construir conocimientos de forma participativa, abierta, horizontal y colectiva y como un espacio deliberativo, interpretativo y de producción cultural y mediática desde postulados alternativos. Entre todas estas dimensiones, la vertiente formativa resulta especialmente relevante. La podcastfera independiente es un espacio donde los productores adquieren competencias tecnológicas y mediáticas a partir de un compendio de estrategias que se pueden agrupar en cinco modalidades: aprender haciendo, aprender consultando (interactuando con podcasters más experimentados), aprender por imitación (copiar estrategias y técnicas de otros podcasters), aprender experimentando (a través del ensayo-error) y aprender enseñando (mostrando a otros cómo desarrollar este tipo de trabajos). La propia podcastfera funciona, por tanto, como un espacio informal de aprendizaje al proveer estos conocimientos de forma peer-to-peer, desde los veteranos a los noveles, así como desde la propia acción de las asociaciones oficiales del medio. Tal fue el caso de la publicación del libro Podcasting, tú tienes la palabra, obra editada por la Asociación Podcast en 2010, cuyo objetivo era analizar el estado del medio en aquel momento. Estructurado en tres partes, el libro -que se alojó en la Red para su descarga gratuita- aborda la importancia del podcast en la difusión del conocimiento científico, las propias dinámicas productoras del medio, la tecnología vinculada a tales procesos y cuestiones legales relativas a la producción online. En el mismo sentido, esta asociación realizó en 2012 una serie de videotutoriales en su canal de YouTube para formar a los podcasters noveles en las competencias más complejas de adquirir a la hora de realizar sus producciones (elaboración de blogs, alojamiento de programas en servicios de podcasting, colocación de reproductores de podcasts en sitios online, gestión de feeds, etc.).

\subsection{Lógica IDIC}

La amplitud de estrategias narrativas, textualidades y formatos que podemos encontrar en el podcasting nos lleva a conceptualizarlo como un medio que, en palabras de Jenkins (2009), mantiene las dinámicas IDIC (Infinita Diversidad en Infinitas Combinaciones), característica propia de los medios alternativos en los que abundan un amplio rango de temas, de estilos narrativos y de tácticas. Los zines planteaban modelos creativos basados en la abundante combinación -incluso disolución- de géneros así como la adopción de nuevas aproximaciones al contenido, la forma y los procesos productivos, especialmente en aquellas publicaciones donde los lectores adquirían el rol de escritores. Esta variedad de formas de presentación de los contenidos conecta con la abundancia de estrategias estructurales que podemos encontrar en el podcasting a la hora de distribuir sus textualidades en diferentes soportes y de expandir sus relatos utilizando diferentes lenguajes y tácticas. El podcasting independiente y amateur despliega proyectos caracterizados por su multiplicidad de contenidos y plataformas, tanto en entornos web como offline. Canales de YouTube, aplicaciones para dispositivos móviles, libros físicos y electrónicos, eventos presenciales, objetos de merchandising e, incluso, webseries, amueblan los proyectos de estos creadores, expandiendo sus relatos más allá del lenguaje sonoro. Esta diversidad de contenidos y pla- 
taformas, lejos de comportarse de forma aislada, guardan conexiones entre sí desarrollando una gramática lógica y una alta complejidad narrativa, presente en los siguientes modelos definidos por García-Marín (2017):

a. Modelo monotema/multiformato. A partir de un programa central que constituye el corazón del proyecto, se crean diferentes podcasts con la misma temática, pero con formato y duración diferenciados.

b. Modelo de red multitemática. Si el modelo anterior apuesta por la multiplicidad de formatos y duraciones, las redes de podcasts se caracterizan por su amplia variedad temática. Estas redes están construidas por varios programas con contenido diferenciado, muy diverso, y presentados por diferentes podcasters.

c. Extensión mediante lenguajes (audio)visuales. Este modelo conduce a los escuchas desde el podcast convencional hasta el consumo de materiales audiovisuales gracias a la producción de webseries y otros contenidos, tales como ilustraciones de producción propia que acompañan a cada nuevo capítulo del podcast y funcionan como carátulas de estos episodios. Cada uno de estos elementos gráficos y audiovisuales funcionan no sólo como un mero acompañamiento o complemento del contenido sonoro, sino que constituyen materiales con pleno significado.

d. Grupo mediático multiplataforma. En este modelo, un primer podcast inicial desarrolla una compleja expansión hacia otros lenguajes y plataformas originando una sólida agregación de medios -página web, canal de YouTube, apps para dispositivos móviles, etc.- que tratan la misma temática, ofreciendo contenidos de alta calidad, muy próximos al estándar profesional.

e. Podcast derivado (spin-off). Entendemos las producciones spin-off en el podcasting como aquellos programas que surgen de podcasts anteriores con los que guardan algún nexo de unión o elemento en común, ya sea la temática o los presentadores.

f. Modelo de extensión bilingüe. Su fundamento es sencillo: consiste en la grabación de un programa con dos versiones en lenguas diferentes y contenidos distintos, ambas incluidas en el mismo proyecto y realizadas por el mismo equipo de podcasters.

Esta filosofía IDIC demuestra la ausencia de jerarquías en los medios alternativos, tanto analógicos como digitales. Siguiendo a Gauntlett (2011), una parte significativa del disfrute del arte y la creatividad online es que no confía en el paradigma del experto para ser validado ni depende de editores ni gatekeepers para su circulación y distribución. En esta línea, Shirky (2010) defiende que incluso las menos ambiciosas e irrelevantes páginas web reflejan una vívida creatividad que debería ser ampliamente valorada porque sugiere a los futuros creadores que ellos mismos también pueden embarcarse en la realización de estas labores mediáticas sin férreos cánones productivos ni esclavitudes creativas.

\section{Conclusiones y discusión. De la cultura de la participación a la conquista de la influencia}

Existen claras analogías entre zines y podcasting -dos medios cuyos desarrollos distan cuatro décadas- desde su caracterización como medios alternativos. Ambos mantienen relaciones sociales transformadas en cuanto a los roles de usuarios y productores, una organización colectiva y una ausencia de profesionalización en las labores comunicativas, de impresión y publicación. En el marco de los procesos, situamos también una aparente emancipación con respecto a los 
escenarios de comunicación verticales, así como la adopción de un funcionamiento en redes peer to peer. También desde el punto de vista temático, existen evidencias que colocan al podcasting en una clara sintonía con respecto a las lógicas de los medios alternativos. La necesidad de rellenar nichos de contenido aún no cubiertos por los medios tradicionales constituye la razón primera y fundamental para el inicio de la actividad mediática de los podcasters.

Los zines tienen la capacidad de reducir la distancia cultural entre creación y recepción a través de los contenidos personales que constituyen experiencias de la vida diaria. La alta presencia de un tipo de "diarios personales hablados" entre los proyectos de podcasting resulta clave para animar a futuros podcasters a lanzarse a la arena mediática manifestando uno de los grandes valores que se elevan en el mundo de los medios alternativos: sus aparentes pocas barreras para la participación en cualquiera de sus niveles, pasando de una intención representativa a una lógica claramente identitaria. Ambos medios promueven la construcción de redes de apoyo entre creadores y seguidores que, suficientemente engarzadas, constituyen amplias comunidades que proveen los conocimientos necesarios para iniciar y desarrollar esta labor mediática. Tanto en el podcasting amateur e independiente como en el zine, se producen procesos de inteligencia colectiva donde la voz de la comunidad prevalece sobre el paradigma del experto; y la lógica de la educación formal y la profesionalización son reemplazadas por los métodos autodidactas de aprendizaje, la experimentación colectiva y las lógicas maker.

El análisis que conecta la cultura del zine con el podcast nos lleva hacia una conclusión evidente: antes de la llegada de la Web 2.0 ya existían espacios para la cultura de la participación y el desarrollo de medios contraculturales, procesos que han sido recuperados y remediados desde determinadas comunidades y especies mediáticas digitales. El usuario del pasado no representaba un papel totalmente pasivo en su relación con los medios. Con la necesaria voluntad de los propietarios y productores de los medios, en la época de los mass media ya existían procesos de participación tanto a nivel micro (el envío de cartas al director en los periódicos o las llamadas de los oyentes en el caso de la radio) como macro (determinados zines dejaban páginas en blanco para ser escritas por ciudadanos comprometidos, quienes debían enviar sus propuestas a la redacción del medio mientras que, por otro lado, las llamadas radios libres y comunitarias que proliferaron en España a partir de los años 80 permitían a individuos y colectivos tener voz y participar en el debate público).

Podría argumentarse, sin embargo, que la verdadera novedad de lo digital es la mayor posibilidad de alcance e influencia que el ciudadano medio puede obtener al propiciar no solo un escaparate sin precedentes (por su alcance global) para su labor mediática, sino también una mayor facilidad en la producción y distribución de los contenidos de los "sin voz" y una mayor capacidad conectiva entre individuos. Nada más lejos de la realidad. Ni la cultura de la participación ni el empoderamiento comunicacional son construcciones novedosas de los medios digitales del siglo XXI, ni las tecnologías 2.0 empoderan al ciudadano más que los medios analógicos para lograr una mayor participación significativa en la arena mediática. El advenimiento en 2004 de la llamada Web 2.0 trajo consigo la aparición de un sinfín de herramientas tecnológicas y medios digitales e interactivos que le otorgaron al usuario, aparentemente, enormes oportunidades para la explotación de la creatividad y la colaboración a fin de lograr una mayor presencia en el debate público. Desde entonces, apareció una corriente de pensamiento excesivamente celebradora sobre el empoderamiento ciudadano que define una reconfiguración del modelo mediático desde un sistema unidireccional y funcionalista a otro donde la información fluye en múltiples direcciones. Es decir, una transformación desde el modelo predominantemente jerárquico y unidireccional 
de los mass media del siglo XX a un sistema aparentemente más multidireccional, más horizontal y más democrático donde los mensajes circularían también desde las audiencias hasta los medios y desde los ciudadanos hasta los centros de poder. El problema de estas corrientes teóricas estriba en su aproximación tecnocéntrica al determinar que la mera inclusión de la tecnología digital 2.0 conlleva un cambio significativo en los modelos y procesos de la comunicación.

Frente a estas perspectivas, es urgente revisar las teorías de la cultura de la participación para distinguir claramente el mero hecho de tener voz de la capacidad para influir de forma significativa en el discurso que circula en los entornos virtuales (Aparici y García-Marín, 2018). La clave es determinar qué tipo de contenidos generados por los internautas pasan desapercibidos, cuáles constituyen mero ruido o falsa participación y cuáles terminan adquiriendo una verdadera relevancia para sus comunidades, para sus lugares de residencia, sus países o para la sociedad en general. Cuáles saltan de lo meramente expresivo a lo verdaderamente relevante.

Las teorías de la cultura de la participación se centraron en la segunda década del siglo XXI en dos aspectos clave: el acceso y las competencias mediáticas y tecnológicas de los usuarios como barreras a superar para lograr tal participación. En el inicio de la tercera década, emergen nuevos sesgos y variables que determinan los procesos participativos. En una esfera digital donde existe sobreabundancia de información-donde una multitud de ciudadanos alojan contenidos propios-, la capacidad de ser visible marca la diferencia para lograr una significativa relevancia en el discurso público. En definitiva, para ser escuchado. La propia arquitectura de la Web configura uno de los sesgos más importantes. La infraestructura en la que se asienta nos remite a una clara jerarquización del acceso al contenido, que Hindman (2008) denominó googlearquía, concepto que refiere la centralidad que tienen los buscadores en la visibilidad y descubrimiento de la información online. En este sentido, el hipertexto funciona como elemento fundamental: el número de enlaces que apuntan a una web está relacionado con la visibilidad que le otorgan los motores de búsqueda que operan en la Red, de modo que los sitios online más conocidos y que, por tanto, reciben más enlaces tienden a ser más fáciles de encontrar. Esta estructura privilegia a aquellos agentes mediáticos, corporaciones y focos de poder tradicionales que ya tienen amplia presencia fuera de la Red y que forman parte del establishment (los ya empoderados), a la vez que dificulta la visibilidad de las voces ciudadanas menos conocidas offline y que pretenden integrar discursos contraculturales. Se produce así un evidente sesgo de popularidad en la distribución de la atención online (Webster, 2014). La Red ofrece más dificultades que oportunidades a la voz de los desposeídos. Tal y como está configurado hoy, Internet no constituye una quiebra real del modelo predominantemente unidireccional donde unos pocos dominan el relato y una multitud pasa desapercibida. Existen evidencias de que Internet representa una concentración de la atención de los usuarios hacia un pequeño número de individuos, medios, servicios y plataformas que concentran el consumo de una forma al menos tan intensa como la existente en el siglo XX. La Red refuerza las relaciones de poder que siempre existieron.

En los medios digitales y las redes sociales, el usuario es colocado en la tesitura de jugar al like, al retuit, al remix y a compartir los contenidos sesgados y falsos que inundan cada día nuestros espacios virtuales. En la línea expuesta por Neil Postman (1985), somos jugadores seducidos por la promesa de una falsa participación en una partida global donde nuestra voz casi nunca resuena con fuerza, pero donde nuestros actos tienen valor político y económico que rentabilizan las elites tecnológicas a partir de procesos de neoconductismo, siempre bajo la mercantilización de nuestros datos (Lanier, 2018). Es por ello que resulta fundamental repensar la cultura de la participación mediática y las teorías del empodera- 
miento comunicacional. Los medios participativos per se no existen. Lo que realmente existen son los ecosistemas de participación que cada proyecto mediático en particular activa a partir de la combinación de las diferentes variables que conforman su gramática específica de interacción entre creadores y usuarios. En este sentido, si entendemos la producción y la distribución de los medios como lenguajes que son tenidos en cuenta desde el lado de los productores, debemos concebir la participación como otro lenguaje más a fin de construir procesos de comunicación más democráticos y empoderadores (García-Marín y Aparici, 2020). La participación significativa ha de constituirse como una estrategia cultural de los medios, los individuos y los grupos sociales para reinventar el poder ciudadano digital y lograr superar esos procesos de falsa participación para lograr una verdadera democratización del discurso mediático público.

\section{Referencias bibliográficas}

Aparici, R. y García-Marín, D. (2018). Comunicar y educar en el mundo que viene. Barcelona: Gedisa.

Atton, C. (1996). Alternative Literature: a Practical Guide for Librarians. Aldershot: Gower.

Atton, C. (2002). Alternative media. London: SAGE Publications.

Aubrey, C. (1981). Beyond the Free Press: Recent Developments in the Radical Press. En Brian Whitaker, News Limited:Why You Can't Read All About It (pp. 167-176). London: Minority Press Group.

Benkler, Y. (2006). The Wealth of Networks. New Haven and London: Yale University Press.

Berry, R. (2015). Serial and ten years of podcasting: Has the medium grown up? Recuperado el 4 de marzo de 2019 de http://www.lasics.uminho.pt/netstation/?lang=en.

Berry, R. (2018). Just Because You Play a Guitar and Are from Nashville Doesn’t Mean You Are A Country Singer: The Emergence of Medium Identities in Podcasting. En Llinares, D.; Fox, N., y Berry, R. (eds.), Podcasting. New Aural Cultures and Digital Media (pp. 15-33). Cham (Suiza): Palgrave Macmillan.

Bey, H. (1991) T.A.Z.: The Temporary Autonomous Zone, Ontological Anarchy, Poetic Terrorism. Brooklyn, NY: Autonomedia. Bolter, D. y Grusin, R. (2000). Remediation: Understanding new media. Cambridge, MA: MIT Press.

Bonini, T. (2015). The 'Second Age' of podcasting: Reframing Podcasting as a new digital mass medium. Quaderns del CAC, 41, pp. 23-33.

Castells, M. (2012). Redes de indignación y esperanza. Madrid: Alianza Editorial.

Cordeiro, P. (2012). Radio becoming r@dio: Convergence, interactivity and broadcasting trends in perspective. Journal of Audience \& Reception Studies, 9(2), pp. 492-510.

De Lara-González, A. y Del Campo-Cañizares, E. (2018). El podcast como medio de divulgación científica y su capacidad para conectar con la audiencia. Revista Mediterránea de Comunicación, 9(1), pp. 347-359. DOI: https://www.doi. org/10.14198/MEDCOM2018.9.1.15 
Espada, A. E. (2017). Ecosistema radiofónico online en Argentina: un análisis comparativo de los contenidos de los sitios webs de agregadores, podcasters, radios online y offline. Revista de la Asociación Española de Investigación de la Comunicación, 4(8), pp. 54-62. DOI: http://dx.doi.org/10.24137/raeic.4.8.7

Fiske, J. (1991). Understanding Popular Culture. London: Routledge.

Gallego, J. I. (2010). Podcasting: Distribución de contenidos sonoros y nuevas formas de negocio en la empresa radiofónica española. Recuperado el 12 de mayo de 2018 http:// eprints.ucm.es/11205/1/T32070.pdf.

García-Marín, D. (2017). La nueva comunicación sonora. Del podcast al transcasting. En: Aparici, R. y García-Marín, D. (coords.) ¡Sonríe, te están puntuando! Narrativa digital interactiva en la era de Black Mirror (pp. 145-163.) Barcelona: Gedisa.

García-Marín, D. (2019). La radio en pijama. Origen, evolución y ecosistema del podcasting español. Estudios sobre el mensaje periodístico, 25(1), 181-196. DOI: https://doi.org/10.5209/ESMP.63723

García-Marín, D. y Aparici, R. (2020). Voces domesticadas y falsa participación: Anatomía de la interacción en el podcasting transmedia. Comunicar, 63. DOI: https://doi.org/10.3916/C63-2020-09

García-Peinazo, D. (2019). A la parrilla suena mejor: El podcast como estrategia didáctica basada en el ABP para el análisis y la gestión de eventos en la asignatura Historia de la Música. Tercio Creciente, 15, pp. 73-84. DOI: https://dx.doi. org/10.17561/rtc.n15.5

Gauntlett, D. (2011). Making is connecting. The social meaning of creativity, from DIY and knitting to Youtube and Web 2.0. Cambridge, MA: Polity Press.

Gillmor, D. (2004). We the media: Grassroots Journalism by the people, for the people. Boston: O'Reilly Media.

Hancock, D. \& McMurtry, L. (2018). I Know What a Podcast Is: Post-Serial Fiction and Podcast Media Identity. En Llinares, D., Fox, N. y Berry, R. (eds.), Podcasting. New Aural Cultures and Digital Media (pp. 81-105). Cham (Suiza): Palgrave Macmillan.

Hindman, M. (2008). The myth of digital democracy. Princeton: Princeton University Press.

Illich, I. (2002). Deschooling Society. London: Marion Boyars.

Jenkins, H. (2009). Fans, blogueros y videojuegos. La cultura de la colaboración. Barcelona: Paidós.

Jenkins, H., Ford, S. y Green, J. (2015). Cultura Transmedia. La creación de contenido y valor en una cultura en red. Barcelona: Gedisa.

Jenkins, H., Ito, M. y Boyd, D. (2016). Participatory Culture in a Networked Era. Cambridge: Polity Press.

Labelle, B. (2018). Sonic Agency. Sound and Emergent Forms of Resistance. London: Goldsmiths Press.

Lanier, J. (2018). Ten Arguments For Deleting Your Social Media Accounts Right Now. London: The Bodley Head.

Lefebvre, H. (1991). Critique of Everyday Life. Volume I: Introduction. London: Verso. 
Lindgren, M. (2014). RN s Creative Audio Unit-what s that all about? The Conversation. Recuperado el 20 de marzo de 2019 de http://theconversation.com/rns-creative-audio-unit- whats-that-all-about-26454.

Llinares, D. (2018). Podcasting as Liminal Praxis: Aural Mediation, Sound Writing and Identity. En Llinares, D., Fox, N., y Berry, R. (eds.), Podcasting. New Aural Cultures and Digital Media (pp. 123-145). Cham (Suiza): Palgrave Macmillan.

López-Villafranca, P. (2019). Estudio de casos de la acción sonora en la radio pública, RNE, y en la plataforma de podcast del Grupo Prisa en España. Anuario Electrónico de Estudios en Comunicación Social Disertaciones, 12(2), pp. 65-78. DOI: https://doi.org/10.12804/revistas.urosario.edu.co/disertaciones/a.6547

Markman, K. M. (2011). Doing radio, making friends and having fun: Exploring the motivations of independent audio podcasters. New Media \& Society, 14(4), 2012, pp. 547-565. DOI: https://doi.org/10.1177/1461444811420848

Martínez-Costa, P. (2015). Radio y nuevas narrativas: de la crossradio a la transradio. En M. Oliveira, y F. Ribeiro (eds), Radio, sound and Internet (pp. 168-187). Proceedings of Net Station International Conference.

Massarelli, V., Perrotta, M. (2006). Podcasting: A change for listeners, a challenge for broadcasters. En Thiermann, S. (ed.). Relating Radio. communities. Aesthetics. Access. (pp. 267-264). Leipzig: Spector.

McHugh, S. (2016). Oral history and the radio documentary/feature: Introducing the COHRD form. Radio Journal: International Studies in Broadcast \& Audio Media, 10(1), pp. 35-51. DOI: 10.1386/rjao.10.1.35_1

McKay, G. (1996). Senseless Acts of Beauty: Cultures of Resistance since the Sixties. London: Verso.

Millette, M. (2011). Independent Podcasting as a Specific Online Participative Culture: a case of study of Montreal s podcasters. Artículo presentado en la AOIR Conference, Montreal University 10-13 de octubre 2011.

Moreno-Espinosa, P. y Román-San Miguel, A. (2020). Podcasting y periodismo. Del periodismo radiofónico de inmediatez a la información radiofónica de calidad. Estudios sobre el Mensaje Periodístico 26(1), pp. 432-453. DOI: https://dx.doi. org/10.5209/esmp.67303

Ortega, I. (2019). Uso del podcast como recurso didáctico para la mejora de la comprensión auditiva del inglés como segunda lengua (L2). Revista de lenguas para fines específicos, 25(2), pp. 9-25. DOI: http://dx.doi.org/10.20420/rlfe.2019.283 Pérez-Alaejos, M., Pedrero-Esteban, L. M. y Leoz-Aizpuru, A. (2018). La oferta nativa de podcast en la radio comercial española: contenidos, géneros y tendencias, Fonseca Journal ofCommunication, 17, 91-106. DOI: http://dx.doi.org/10.14201/ fjc20181791106

Postman, N. (1985). Amusing Ourselves to Death. Public Discourse in the Age of Show Business. New York: Viking Penguin Inc.

Ramos-Ruiz, A. (2015). Radio hertziana vs. radio en Internet: Un análisis comparativo. Opción, 31(4), pp. 758-774.

Rheingold, H. (2000). The Virtual Community. Cambridge, MA: MIT Press.

Rodríguez-Pallarés, M. (2017) Reutilización de la ficción sonora en la Cadena Ser. El caso de Podium Podcast. Área Abierta. Revista de comunicación audiovisual y publicitaria 17(1), pp. 83-98. DOI: http://dx.doi.org/10.5209/ARAB.53445

Sellas, T. (2011). El podcasting: La (r)evolución sonora. Barcelona: Universidad Oberta de Catalunya. 
Sellas, T. y Solà, S. (2019). Podium Podcast and the freedom of podcasting: Beyond the limits of radio programming and production constraints. Radio journal international studies in broadcast \& audio media, 17(1), pp. 63-81. DOI: https://doi. org/10.1386/rjao.17.1.63_1

Shirky, C. (2010). Cognitive Surplus. How Technology Makes Consumers into Collaborators. New York: Penguin Books. Silverstone, R. (1999). Why Study the Media. London: Sage.

Spinelli, M. y Dann, L. (2019). Podcasting. The Audio Media Revolution. New York: Bloomsbury.

Sullivan, J. L. (2018). Podcast Movement: Aspirational Labour and the Formalisation of Podcasting as a Cultural Industry. En Llinares, D., Fox, N. y Berry, R. (eds.), Podcasting. New Aural Cultures and Digital Media (pp. 35-56). Cham (Suiza): Palgrave Macmillan.

Swiatek, L. (2018). The Podcast as an Intimate Bridging Medium. En Llinares, D., Fox, N., y Berry, R. (eds.), Podcasting. New Aural Cultures and Digital Media (pp. 173-187). Cham (Suiza): Palgrave Macmillan.

Webster, J. G. (2014). The marketplace of attention. How audiences take shape in a digital age. Cambridge, Massachusetts: MIT Press.

Wilson, R. Z. (2018). Welcome to the World of Wandercast: Podcast as Participatory Performance and Environmental Exploration. En Llinares, D., Fox, N. y Berry, R. (eds.), Podcasting. New Aural Cultures and Digital Media (pp. 273-298). Cham (Suiza): Palgrave Macmillan.

Wrather, K. (2016). Making "Maximum Fun” for fans: Examining podcast listener participation online. The Radio Journal-International Studies in Broadcast \& Audio Media, 14(1), 2016, pp. 43-63. DOI: 10.1386/rjao.14.1.43_1 